\title{
Strongyloides stercoralis infestation in an onco-haematogenic patient
}

\author{
Carlotta Guidetti', Francesco Merli², Luigi Vecchia' \\ I U.O. di Microbiologia, Arcispedale Santa Maria Nuova Reggio Emilia \\ 2 U.O. di Ematologia, Arcispedale Santa Maria Nuova Reggio Emilia
}

Key words: Strongyloides stercoralis, lymphoma, hypereosinophilia.

Infestazione da Strongyloides stercoralis in paziente oncoematologico

SUMMARY

We describe a case of an infection caused by Strongyloides stercoralis in a patient affected by onco-haematological disease, who manifested an high peripheral hypereosinophilia after a few cycles of specific chemotherapy.

\section{INTRODUZIONE}

La strongiloidiasi è una malattia parassitaria sostenuta dal nematode Strongyloides stercoralis. L'infestazione avviene sempre per contatto diretto della cute integra con terreno contaminato da larve filariformi infestanti (larve L3). Queste ultime penetrano attraverso la cute e migrano tramite la circolazione linfatica e venosa al cuore destro e poi ai polmoni; da qui negli alveoli polmonari per risalire l'albero tracheobronchiale sino al laringe, per essere infine ingerite e raggiungere l'apparato intestinale, ove maturano in femmine adulte che producono uova per partenogenesi (13). Le uova prodotte dalle femmine passano molto rapidamente dalla fase di morula a quella embrionata e la schiusa è talmente rapida che nei campioni fecali del paziente infestato vengono reperite solo le larve rabditoidi, come tale non infestanti (larve L1). Il periodo di prepatenza nell'uomo è di circa 1 mese (a volte meno). Esiste anche l'auto-infestazione, che è tipica di questo elminta (14): alcune larve rabditoidi possono già maturare a larve filariformi in sede intestinale (auto-infestazione interna) oppure maturare in sede perianale e penetrare la cute circostante con la possibile "sindrome della larva currens" (autoinfestazione esterna); da qui il medesimo ciclo. L'età avanzata e/o vari fattori di immunodepressione sono in genere alla base delle autoinfestazioni $(5,7)$.

Il meccanismo patogenetico di S. stercoralis è attribuibile al suo peculiare ciclo biologico grazie al quale il nematode può persistere senza manifestazioni cliniche per un tempo indefinito nell'ospite infestato (7). L'infestazione diventa quindi cronica (11), ma non necessariamente sintomatica (in caso affermativo si manifesta solitamente con disturbi intestinali aspecifici e/o ipereosinifilia periferica, peraltro non necessariamente sempre presente). Nelle forme più evidenti l'infestazione è in grado di produrre alterazioni anatomiche - tissutali e perivasali a livello polmonare, danni istopatologici a livello intestinale e di attivare una risposta immunitaria che si manifesta con ipereosinofilia periferica anche elevata (5). L'infestazione da $S$. stercoralis è descritta in tutti i continenti. Predilige i paesi tropicali e sub-tropicali, ma è presente anche in regioni a clima temperato, Italia compresa $(1,3,4$, $12,15)$. La maggior parte dei soggetti infestati in zone temperate presenta un'età media elevata (in genere superiore ai 60 anni) e un passato di attività agricole a rischio (lavoro in risaie, aratura o vangatura dei campi con metodi manuali e simili, con la conferma anamnestica di aver camminato a "piedi scalzi" in terreni non asciutti o secchi), come confer- mato, per esempio, da alcuni studi di prevalenza nelle aree di Pavia e di Novara (10).

\section{CASO CLINICO}

Il paziente è un uomo di 71 anni, reggiano, che viene sottoposto a 6 cicli di chemioterapia per "Linfoma non Hodgkin diffuso a grandi cellule B", inquadrato come "stadio II E A" per interessamento della tonsilla palatina sinistra e di alcuni linfonodi latero-cervicali omolaterali, in assenza di sintomi sistemici. Agli esami di routine eseguiti prima della chemioterapia viene rilevata una ipereosinofilia del $22 \%$, considerata inizialmente come secondaria al linfoma. Durante la chemioterapia, compare una broncopneumopatia cronica enfisematosa, un eritema a livello sternale, lieve febbricola, astenia, tosse secca, insonnia, diarrea e l' ipereosinofilia sale al 50\%. Viene esclusa l'infezione da CMV e una sindrome ipereosinofila, mentre all'esame coproparassitologico vengono repertate larve rabditoidi di $S$. stercoralis in seguito all'osservazione al microscopio ottico dopo concentrazione delle feci con formolo - etilacetato (Figura I). La diagnosi di strongiloidiasi è stata successivamente confermata mediante esame colturale del campione che ha consentito di evidenziare all'osservazione microscopica larve filarioidi (Figura II) e forme adulte del parassita (Figura III e IV). L'esame colturale è stato eseguito distribuendo circa due grammi di feci su una piastra di terreno cled-agar e incubata a $35^{\circ} \mathrm{C}$, al buio. Dopo 3 giorni sono stati aggiunti alla piastra alcuni cc di formalina al $10 \%$, il liquido è stato raccolto in provetta, centrifugato per cinque minuti a 1100 g; è stato infine allestito un vetrino e osservato al microscopio ottico. All'anamnesi il paziente riferisce di aver camminato per anni a piedi nudi per le campagne reggiane dove aiutava il padre in lavori agricoli; riferisce, inoltre, da circa 40 anni, episodi frequenti di nausea e alternanza di periodi di stipsi a periodi di diarrea profusa. Il clinico prescrive al paziente terapia con albendazolo $400 \mathrm{mg}$ per 7 giorni. Dopo terapia, l'esame delle feci rivela ancora la presenza di larve di S. stercoralis. Si è reso così necessario recuperare un farmaco (ivermectina), non in commercio in Italia, somministrato per 4 giorni in dose di $200 \mathrm{microgr} / \mathrm{Kg} / \mathrm{die}$, grazie al quale il paziente ha risolto l'infestazione e ha completato i cicli di chemioterapia con ottenimento della remissione completa del linfoma. Inoltre, per la prima volta da molti anni, l'alvo è divenuto regolare e si mantiene tale ad un anno dal termine della terapia.

\section{Corresponding author: Carlotta Guidetti}

Laboratorio di Microbiologia, Arcispedale Santa Maria Nuova di Reggio Emilia

42 100 Reggio Emilia, Via Risorgimento 80 - Tel: 3498466603

E.mail: carlottaguidetti@libero.it 


\section{DISCUSSIONE}

Il caso qui riportato suggerisce l'importanza della ricerca di $S$. stercoralis quando ci si trovi di fronte ad una ipereosinofilia di natura non determinata o ad un'anamnesi remota sospetta (attività agricole a rischio) nei sempre più numerosi soggetti immunodepressi, o semplicemente anziani. Per la ricerca mirata S.stercoralis, spesso non è sufficiente un normale esame coproparassitologico standard, ma sono necessarie indagini supplementari: i due principali sistemi raccomandati in letteratura sono il "Metodo di Baermann" e la "coltura su terreno specifico" cercando di analizzare sempre 3 campioni emessi a giorni alterni (6). Tale ricerca è fondamentale prima di ogni terapia cortisonica o antiblastica per evitare l'insorgenza di complicanze in soggetti già affetti da gravi patologie e/o in attesa di trapianto (2). La strongiloidiasi, infatti, può decorrere per moltissimi anni in modo asintomatico o paucisintomatico (9) in quanto nel soggetto parassitato si instaura una sorta di equilibrio tra parassita e ospite, tale da non provocare danni apparenti. Ma allorché subentrano fattori di immunocompromissione, i medesimi alterano questo precario equilibrio, che si sbilancia a favore del parassita, favorendone la riattivazione. Se, in particolare, viene somministrata una terapia cortisonica, S. stercoralis inizia a moltiplicarsi anche a dismisura, sovvertendo qualsivoglia meccanismo di controllo, con la conseguente possibilità di esito infausto per il soggetto, in genere come conseguenza di una grave sepsi da batteri Gram negativi (8).

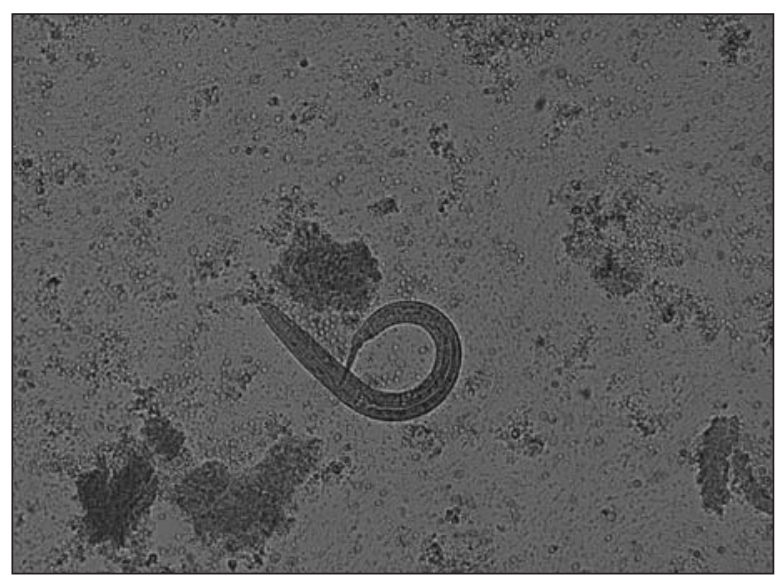

Figura I. Larva rabditoide di Strongyloides stercoralis $(20 \times 10)$ osservata al MO dopo FEA; si distinguono la cavità buccale corta e le due strozzature a livello esofageo.

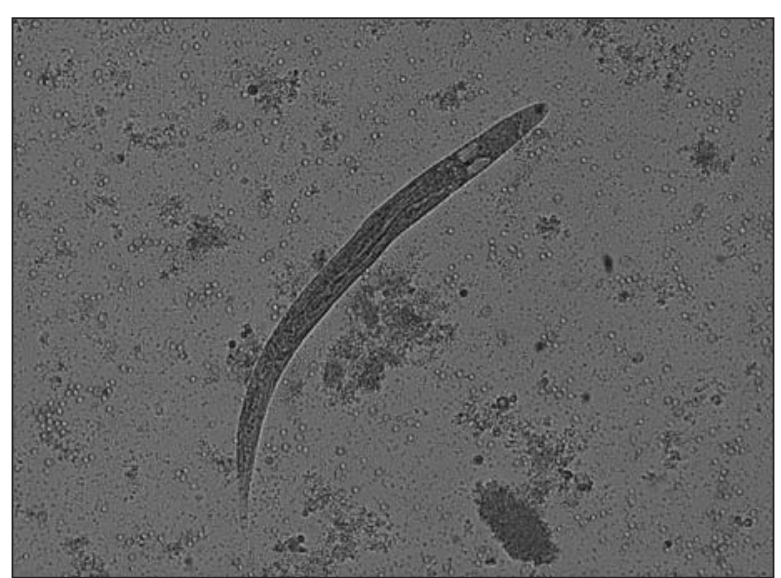

Figura II. Larva filariode di Strongyloides stercoralis $(20 \times 10)$ osservata al MO dopo esame colturale; la cavità buccale si riduce rispetto alle LI e non sono presenti strozzature a livello esofageo.

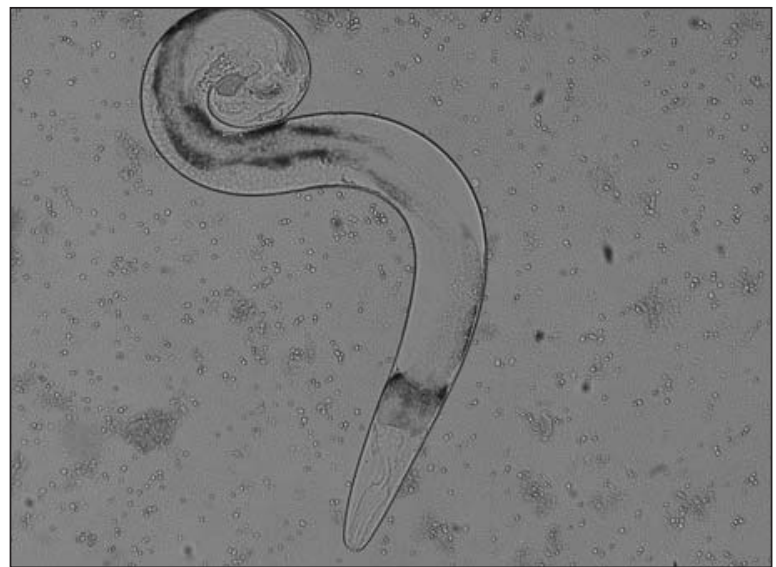

Figura III. Adulto maschio di Strongyloides stercoralis $(10 \times 10)$ osservato al MO dopo esame colturale caratterizzato dall'estremità caudale ricurva su se stessa.

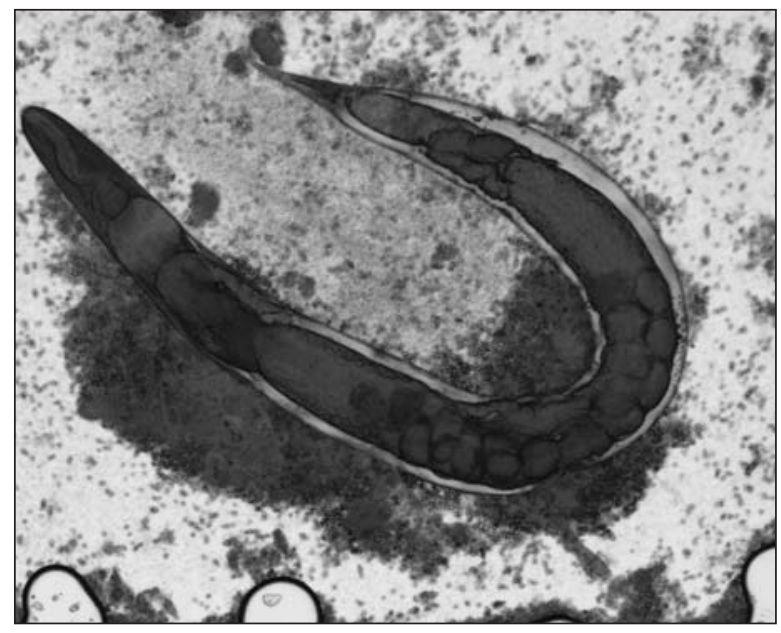

Figura IV. Femmina partenogenetica di Strongyloides stercoralis ( 10 $X$ 10) osservata al MO dopo esame colturale; l'estremità caudale è affilata e sono visibili le uova nella cavità uterina.

\section{Ringraziamenti}

Si ringrazia il dr. Daniele Crotti, di Perugia, per lo stimolo dato alla stesura del presente articolo e per il supporto fornito per la sua revisione.

\section{BIBLIOGRAFIA}

1. Adams M, Page W, Speare R. Strongyloidiasis: an issue in Aboriginal communities. Rural and Remore Health. Journal 3 (online) 2003; 152 Available at: http://rrh.deakin.edu.au

2. Barozzi G, Pinelli G, Varani M, et al. Considerazioni cliniche su un caso di iperinfezione da Strongyloides stercoralis. Rass Pat App Respirat 2002; 17 : 214-8.

3. Bernieri F, Galli D, Giordano S, et al. Indagine nazionale AMCLI-CoSP sulla diffusione delle parassitosi intestinali. Microbiol Med 1996; 11(4): 463-72.

4. Cox FEG. History of Human Parasitology. Clinical Microbiology Reviews 2002; 15(4): 595-612.

5. Crotti D, D’Annibale ML, Rossi S. Strongyloides stercoralis e strongiloidiasi in Umbria: analisi di una casistica decennale. Microbiol. Med. 2005; 20: $250-256$.

6. Crotti D. Le parassitosi intestinalied uro-genitali conoscenze di base e indicazioni diagnostiche. Caleidoscopio Italiano 2006 Ed. Selecta medica.

7. Gill GV, Beeching NJ, Khoo S, et al. ABritish Second World War veteran with disseminated strongyloidiasis.Trans R Soc Trop Med Hyg 2004; 98 : 382-6.

8. Keiser PB, Nutman TB. Strongyloides stercoralis in Immunocompromised Population. Clinical Microbiology Reviews 2004; 17(1): 208-17.

9. Mansfield LS, Niamatali S, Bhopale V, et al.Strongyloides stercoralis: maintenance of exceedingly chronic infections. Am J Trop Med Hyg 1996; 55(6): 617-24. 
10. Pirisi M, Salvador E, Bisoffi Z, Gobbo M, Smirne C, Gigli C, Minisini R, Fortina G, Bellomo G, Batoli E. Unsuspected strongyloidiasis in hospitalized elderly patients with and without eosinophilia. Clin Micr and Infect 2006; 12:787-792. 370. 48.

11. Ricci L., Barbieri Palmieri F. Strongiloidiasi disseminata: descrizione di due casi. Giorn. It. Mal.Inf. 1997; 3: 112-114.

12. Roman SP, Guzman AP, Guillen SM et al. Edemic strongyloidiasis on the Spanish Mediterranean coast. Q J Med 2001; 94: 357-63.
13. Siddiqui AA, Berk SL. Strongyloidiasis. Current Treatment Options in Infectious Diseases 2003; 5: 283-9.

14. Sing A, Leitritz L, Bogner JR, Heesemann J. First-Glance Diagnosis of Strongyloides stercoralis Autoinfection by Stool Microscopy. J Clin Microbiol 1999; 37(5): 1610-1.

15. Tzanetou K, Geros I, Kalogeropoulou E et al. Is Strongyloides stercoralis endemic in Greece Arch Hellen Med 2003; 20(1): 60-6. 